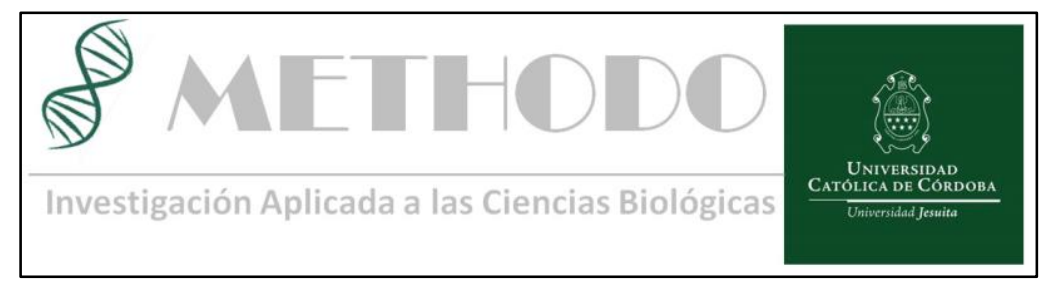

\title{
Resecciones multiviscerales en el Tratamiento Quirúrgico de las Recaídas Locoregionales de Cirugía de RectoSigma. Factibilidad, Complicaciones y Resultados Oncológicos
}

\author{
Multivisceral Resections in the Surgical Treatment of Locoregional \\ Relapses of RectoSigma Surgery. Feasibility, Complications and \\ Oncologic Results
}

\begin{abstract}
Mandojana, F; Pedraza Zalazar, I; Pairola, A; Brancato, F; Sánchez Loria, F; Huertas, E (*).
Las inmunodeficiencias primarias (IDP) son enfermedades de baja prevalencia. Existen signos de alarma para su sospecha clínica. Los objetivos fueron describir características clínicas y signos de alarma de pacientes con IDP y comparar diferencias clínicas entre déficit selectivo de inmunoglobulina A $(\lg A)$ y otras IDP. Se estudiaron 89 pacientes, con mediana de edad, al momento del diagnóstico, de 6 años $(4,08-11,67) .53(59,5 \%)$ pacientes, varones. $54(60,7 \%)$ pacientes, con déficit selectivo de $\operatorname{lgA}$, y 35 $(39,3 \%)$, con otras IDP. Las principales manifestaciones clínicas fueron rinofaringitis en $65(73,03 \%)$ pacientes y atopia en $39(43,82 \%)$ pacientes. Presentaron signos de alarma $24(26,97 \%)$ pacientes, ninguno con déficit selectivo de IgA. En los pacientes con otras IDP, se observó mayor incidencia de infección respiratoria baja, sepsis, infecciones cutáneas, candidiasis mucocutánea, alteraciones dentales, malformaciones cardiovasculares, angioedema, internaciones y mortalidad. $10(28,57 \%)$ pacientes recibieron gammaglobulina endovenosa; 15 (42,85\%), antibiótico-profilaxis; y 2 (2,24\%), antimicóticoprofilaxis. Palabras clave: infecciones respiratorias, hipersensibilidad, gammaglobulinas, síndromes de inmunodeficiencia, deficiencia de $\lg A$.
\end{abstract}

Palabras claves: infecciones respiratorias; hipersensibilidad; gammaglobulinas; síndromes de inmunodeficiencia; deficiencia de $\lg \mathrm{A}$.

Primary immunodeficiencies (PID) are low-prevalence diseases. There are warning signs that may raise clinical suspicion. The objectives of this study were to describe the clinical characteristics and warning signs of patients with PID and to compare the clinical differences between selective immunoglobulin A $(\lg A)$ deficiency and other PIDs. Eighty-nine patients were studied; their median age at the time of diagnosis was 6 years old (4.08-11.67). Fifty-three (59.5\%) patients were male. Fifty-four (60.7\%) patients had selective IgA deficiency, and $35(39.3 \%)$ had other PIDs. The main clinical manifestations were rhinopharyngitis in $65(73.03 \%)$ patients and atopy in 39 (43.82\%). Twenty- four (26.97\%) patients showed warning signs, and none had selective $\lg A$ deficiency. Patients with other PIDs had a higher incidence of lower respiratory tract infection, sepsis, skin infections, mucocutaneous candidiasis, dental alterations, cardiovascular malformations, angioedema, hospitalizations and death. Ten (28.57\%) patients received intravenous gammaglobulin, 15 (42.85\%) antibiotic prophylaxis, and 2 (2.24\%) antifungal prophylaxis.

Keywords: respiratory infections; hypersensitivity; gamma globulin; immune deficiency syndromes, IgA deficiency

(*) Servicio de Oncología Quirúrgica del Instituto Alexander Fleming, Buenos Aires. Argentina. Correspondencia: Mandojana, Facundo (fmandojana@yahoo.com)

Revista Methodo: Investigación Aplicada a las Ciencias Biológicas. Facultad de Medicina. Universidad Católica de Córdoba. Jacinto Ríos 571 Bo Gral. Paz. X5004FXS. Córdoba. Argentina. Tel.: (54) 351 4517299 / Correo: methodo@ucc.edu.ar / Web: methodo.ucc.edu.ar | ARTICULO ORIGINAL 


\section{Introducción}

El cáncer colorectal constituye una de las principales causas de muerte por cáncer en el mundo occidental. Específicamente el cáncer de recto constituye aproximadamente un tercio de los casos y su resección quirúrgica es la principal opción curativa para la enfermedad primaria ${ }^{1-2}$.

A pesar de los grandes avances actuales en el tratamiento multidisciplinario de estos pacientes la tasa de recaída local observada en el promedio de los centros generales y hospitales de la comunidad oscila desde $5.3 \%$ hasta el $20-22 \%$ constituyendo entonces un problema con una incidencia significativa en la práctica oncológica que habitualmente resulta de difícil resolución, por lo complejo de las resecciones quirúrgicas que implica ${ }^{2-5}$.

\section{Objetivo}

Revisar la experiencia de un solo centro con 57 resecciones multiviscerales en pacientes con $\mathrm{Ca}$ de rectosigma recaído para determinar la factibilidad técnica, revisando las complicaciones y evaluando el tiempo a la progresión y la sobrevida en estos pacientes.

\section{Material y métodos}

Hemos llevado a cabo una revisión retrospectiva sistemática de las historias clínicas de todos los pacientes operados por $\mathrm{Ca}$ de RectoSigma Recaído en el Instituto Alexander Fleming entre octubre de 1994 y diciembre de 2014. Fueron elegibles los pacientes operados en la Institución con recurrencia locoregional por $\mathrm{Ca}$ de Recto o de Rectosigma que requirieron una resección multivisceral.

Todos los pacientes fueron estudiados preoperatoriamente con evaluación prequirúrgica clínica de rutina y se les realizó TAC de tórax, abdomen y pelvis y eventual RNM detallada de la pelvis para evaluar resecabilidad.

En algunos pacientes y en forma selectiva por estudios dudosos se utilizó PET para mejorar la estatificación evaluando la presencia de enfermedad a distancia.

De un total de 176 pacientes operados por recaídas pelvianas por ca de recto sigma, 57 cumplieron los requisitos de inclusión, con edades al momento del rescate quirúrgico entre 42 y 76 años (mediana 59,6 años), (54.4\%) de sexo masculino.

Se analizó la factibilidad de la cirugía, los márgenes quirúrgicos de resección, el tiempo de cirugía, la estadía hospitalaria y las complicaciones postoperatorias.

La mortalidad perioperatoria se definió como aquella ocurrida dentro de los 30 días PO. Se definió el tiempo a la progresión como aquel que va desde el rescate quirúrgico a la reaparición de enfermedad ya sea local o a distancia.

En lo que respecta al análisis de sobrevida se evaluó la SG (sobrevida global) desde el diagnóstico inicial de la enfermedad y la SE (sobrevida específica) desde el rescate quirúrgico multivisceral hasta el evento muerte o pérdida del paciente en seguimiento.

\section{Resultados}

El sitio de recaída inicial fue locoregional en los 57 casos analizados. El dolor fue el síntoma más frecuente en la recaída, seguido por la mucorrea y el sangrado.

La resecciones atípicas multiviscerales en bloc objeto de este análisis incluyeron vejiga, útero $y$ anexos, próstata, intestino delgado, pared abdominal, hígado, ganglionar iliaca o sacro. Debido a la selección de los casos se accedió a resección R0 en el $89 \%$ de los casos de esta serie. (51/57).

La duración mediana de la cirugía fue de 4.12 hs ( $R=1.5-8 \mathrm{hs})$ y el tiempo mediano de internación fue de 11 días $(R=4-30$ d) 31 pacientes presentaron algún tipo de complicación 
perioperatoria, constituyendo una morbilidad global de la serie del $54.3 \%$.

Las complicaciones más frecuentes fueron los abscesos pelvianos profundos (21\%), las infecciones de herida quirúrgica con un $21 \%$, seguidas por las fístulas (rectovaginales, rectovesicales, urinarias, etc.) que correspondieron al $18.5 \%$, y el ileo postoperatorio prolongado en el $16 \%$. Se registraron 37 complicaciones en esos 31 pacientes según se registra en la tabla siguiente:

\begin{tabular}{|l|l|}
\hline Complicación & $\mathbf{N}(\%)$ \\
\hline Fístulas (R-Vag; R-Ves; Urinarias, etc.) & $7(18.4 \%)$ \\
\hline Abscesos profundos (pelvianos) & $8(21 \%)$ \\
\hline Infeccion de herida abdominal & $8(21 \%)$ \\
\hline Reoperaciones & $1(2.6 \%)$ \\
\hline Hemoperitoneo & $3(7.8 \%)$ \\
\hline Dehiscencia via kraske & $2(5.2 \%)$ \\
\hline Ileo & $6(15.7 \%)$ \\
\hline Ulcera duodenal perforada & $1(2.6 \%)$ \\
\hline Arritmia & $1(2.6 \%)$ \\
\hline
\end{tabular}

No hubo mortalidad perioperatoria en esta serie. El tiempo a la progresión (TTP) mediano fue de 11 meses (rango entre 2-48 meses) Para este análisis se excluyeron los 5 pacientes con resecciones R1 $(n=5)$ y $R 2(n=1)$.

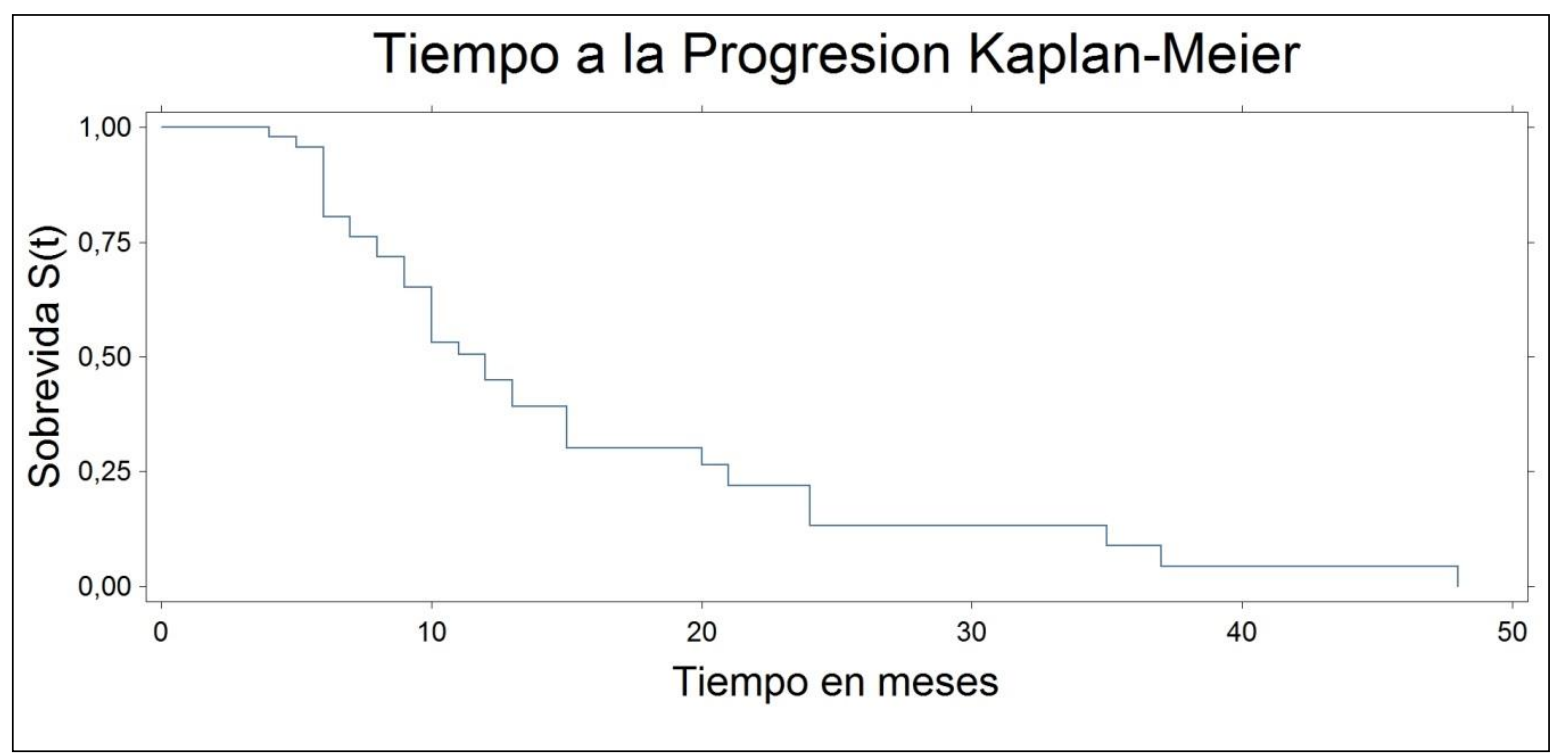

Revista Methodo: Investigación Aplicada a las Ciencias Biológicas. Facultad de Medicina. Universidad Católica de Córdoba. Jacinto Ríos 571 Bo Gral. Paz. X5004FXS. Córdoba. Argentina. Tel.: (54) 351 4517299 / Correo: methodo@ucc.edu.ar / Web: methodo.ucc.edu.ar | ARTICULO ORIGINAL 
En lo que respecta al análisis de sobrevida (resultados oncológicos) se evaluó la sobrevida global medida desde el diagnóstico de la enfermedad y la sobrevida específica medida desde el rescate quirúrgico multivisceral.
La sobrevida específica mediana resultó de 16 meses ( $R=1-215$ meses). La sobrevida global mediana para los 57 pacientes resultó de 36 meses $(R=$ $7-224$ meses) con un $21 \%$ a 5 años. Al analizar sólo los que accedieron a resección R0, dicho valor se incrementó al $38 \%$ a 5 años.
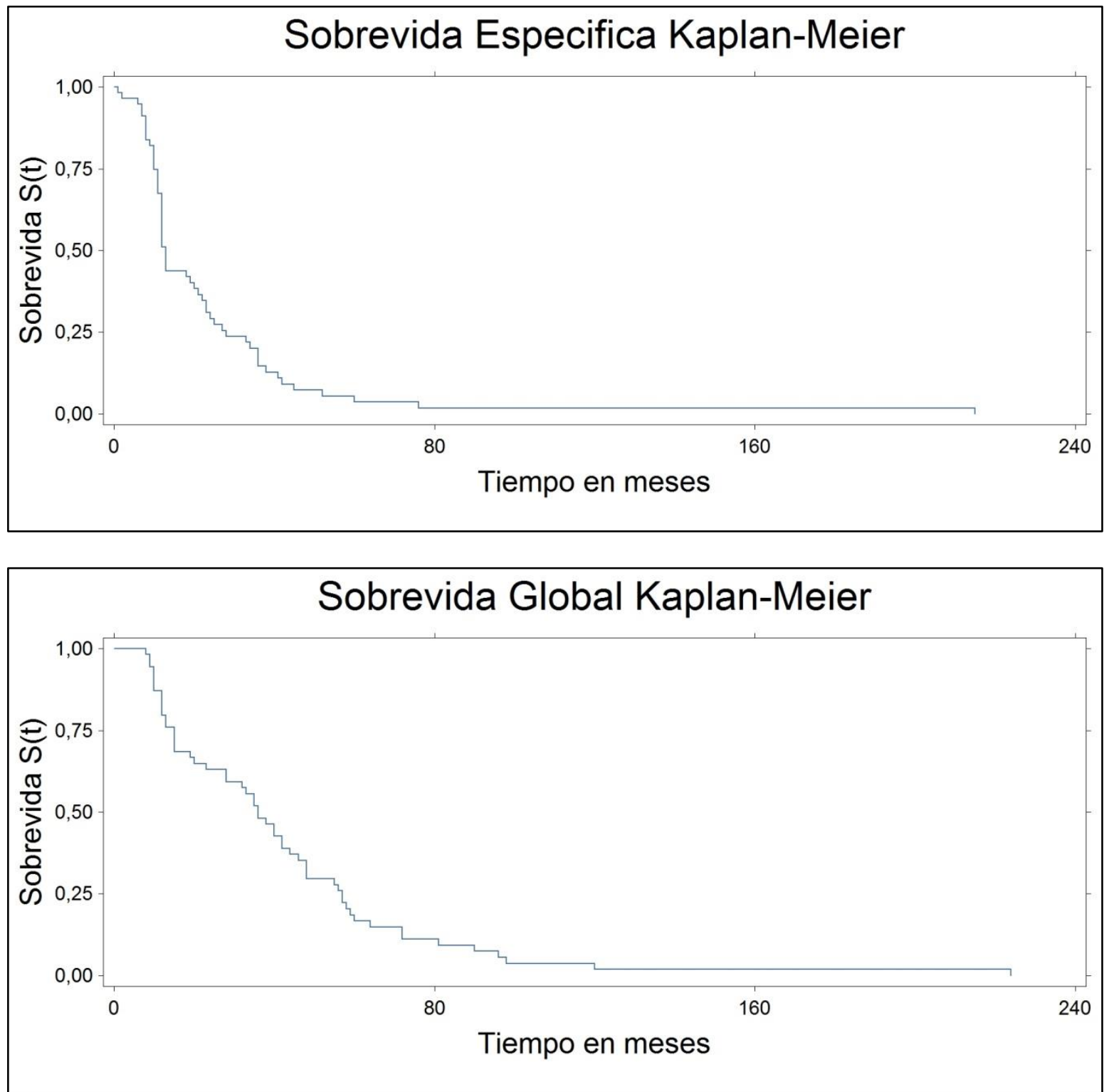


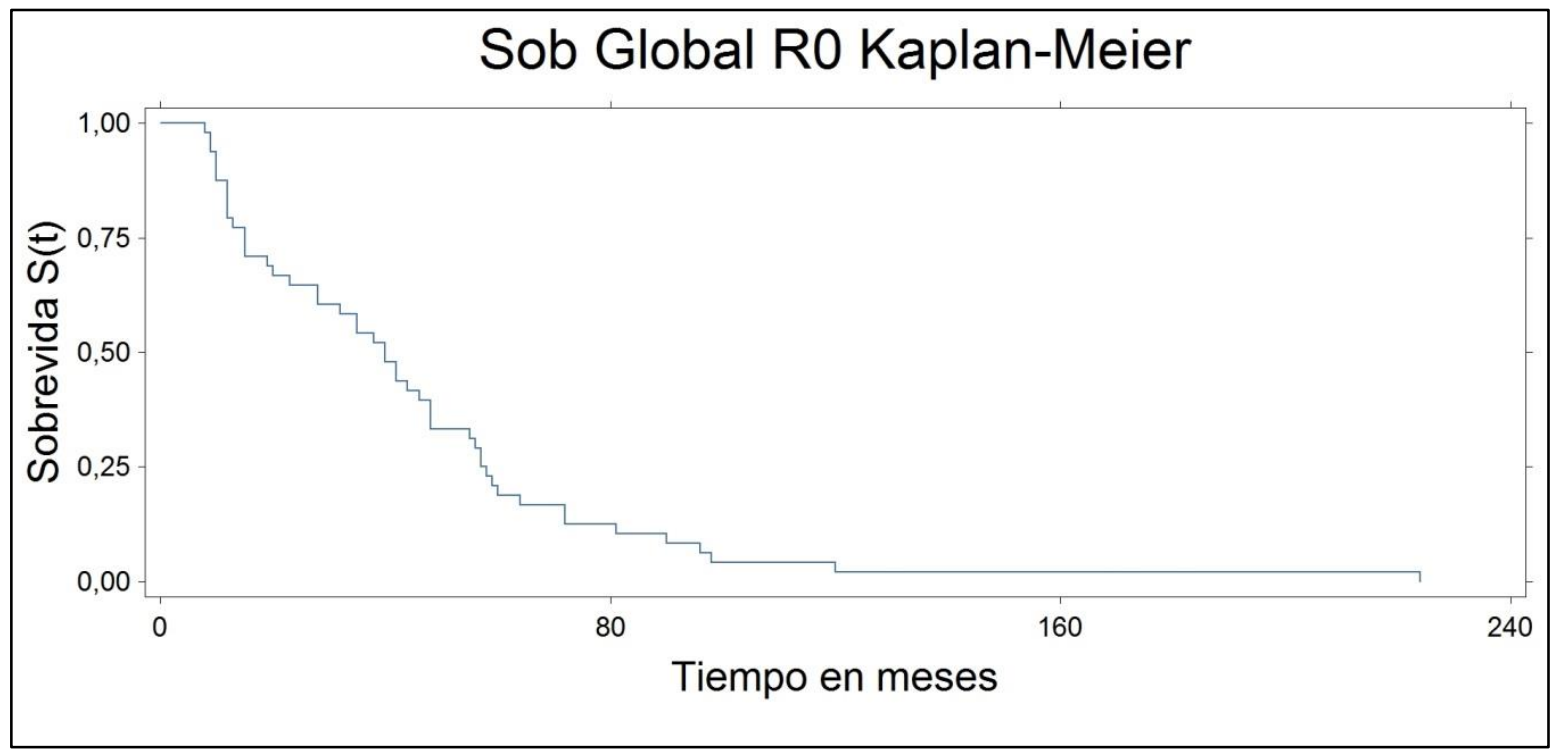

\section{Discusión}

Las recaídas locales son un problema relativamente frecuente en el cáncer colorectal, oscilando en las series históricas quirúrgicas entre el 20 $y$ el $40 \%$ de los casos $^{3}$. Con las modernas técnicas quirúrgicas y con la administración de nuevas estrategias terapéuticas con quimioterapia y/o radioterapia, la incidencia se ha reducido a menos del $10 \%{ }^{5}$.

La recaída local es una situación de difícil manejo. El abordaje terapéutico debe ser siempre contemplado dentro de un equipo multidisciplinario. Sólo cuando se diagnostica precozmente puede ser resecable.

Para los pacientes con recaída local aislada después de un intento inicial de resección curativa se debe intentar un rescate quirúrgico agresivo que puede incluir la resección abdominoperineal o exenteración pélvica total. Si la resección radical es posible se puede obtener un largo período de supervivencia libre de enfermedad8.

El uso de la quimiorradioterapia en pacientes no irradiados previamente con recaídas pélvicas locales localmente avanzadas (invasión de la pared pélvica, el sacro, o los órganos adyacentes), puede aumentar la posibilidad de resección ${ }^{2-5-6}$.

La radioterapia intraoperatoria en aquellos pacientes con recaída local que ya han recibido RT externa previamente, podría mejorar el control local con morbilidad aceptable. Otra posibilidad en estos pacientes seria intentar una neoadyuvancia con el esquema más activo posible con la finalidad de obtener una reducción del tumor previo a la cirugía de rescate $e^{5-7}$.

La cirugía es actualmente la única opción curativa que se les puede ofrecer a los pacientes con $\mathrm{RL}$ por cáncer de recto, alcanzando sobrevidas de hasta el $50 \%$ a 5 años con adecuado control local ${ }^{4}$.

La resección multivisceral de los tumores primarios avanzados de colon o recto está ampliamente avalada en la bibliografía en pacientes bien seleccionados, no obstante ese no es el caso en las recaídas locorregionales cuya resección curativa todavía es considerada por algunos profesionales como riesgosa e inútil y más aún cuando implica resecciones multiviscerales complejas ${ }^{3-8-9}$.

La cirugía de las recaídas pelvianas de cáncer colorrectal es de por sí compleja y presenta eventuales complicaciones intra y postoperatorias reportadas en las distintas series publicadas hasta de un $42-50 \%$. En 
nuestra serie la morbilidad fue similar resultando del $54 \%{ }^{4-6}$.

Estas complicaciones constituyen un verdadero desafío y a la vez pueden condicionar la selección de los candidatos a dicho procedimiento. Por estos motivos el trabajo multidisciplinario resulta insustituible en la selección y el ulterior soporte de estos pacientes, el cual puede en muchos casos extenderse por largos períodos.

Las resecciones multiviscerales se han incrementado en los últimos años y han sobrepasado el $10 \%$ en el caso de los tumores primarios avanzados. En el caso de las recaídas locoregionales, la proporción es mayor por la complejidad anatómica de estos casos, y aunque aumentan la morbilidad y la mortalidad, constituyen una opción terapéutica eficiente en la prolongación de la sobrevida y mejora de la calidad de vida de estos complejos pacientes ${ }^{1-8-9}$.

La cirugía multivisceral (vejiga, uréteres, ginecológica, intestinal, vascular y partes blandas) requiere una exigente formación técnica al cirujano actuante para poder resolver las distintas situaciones durante el acto quirúrgico ${ }^{1-2-9}$.

Si bien en general el equipo tratante cuenta con imágenes preoperatorias de la mejor calidad disponible, en general la decisión de realizar una resección multivisceral y que órganos va a incluir la misma se toma durante el procedimiento quirúrgico ya que lo que las imágenes en ocasiones no aclaran suficientemente son diferencias milimétricas que pueden implicar la conservación o no de un órgano (ej. Vejiga o recto) que si se resecan completamente ocasionan secuelas mutilantes definitivas que empeoran marcadamente la calidad de vida del paciente ${ }^{6}$.

En una proporción significativa (entre 30 y $45 \%$ de los casos según distintas series) lo que se evalúa intraoperatoriamente como infiltración tumoral resulta en la anatomía patológica ser proceso inflamatorio y fibrosis. Sin embargo surge claramente de las distintas publicaciones tanto en recaídas como en tumores primarios avanzados que la separación quirúrgica de dichas adherencias redundan en tasas más elevadas de recaída local y peor sobrevida libre de enfermedad y global $^{5-6}$

La resección $\mathrm{R} 0$ es el objetivo fundamental en estas operaciones. La ampliación de la cirugía se define intraoperatoriamente para evitar violar las interfases tumorales. Los factores pronósticos que atentan contra la posibilidad de resección Ro han sido expuestos en la literatura y son: Género masculino, edad avanzada a la recaída, Miles inicial, tumor de alto grado, estadio alto de Wanebo en la recurrencia, recaida extraluminal, hidronefrosis bilateral, rápido aumento del CEA, dolor ciático, más de dos sitios de fijación a la pared pelviana, $\mathrm{BMI}>30$ y cirugía llevada a cabo fuera de centros terciarios.

Muchos de estos datos, si bien disponibles en nuestra serie, resultan difíciles de correlacionar debido al tamaño exiguo de la muestra.

En lo que a sobrevida se refiere, las series más recientes publicadas muestran valores del $20-40 \%$ a 5 años con una mediana de 24-32 meses. Pero si se incluyen solo las resecciones R0 el valor se aproxima más al $40 \%$, por lo que queda claro que más allá de los parámetros citados en el párrafo anterior la resección $\mathrm{RO}$ es el factor predictivo independiente principal para sobrevida global y libre de enfermedad $^{5}$.

Nuestro set de pacientes mostró una sobrevida global del $21 \%$ a 5 años para la serie global y del $38 \%$ a 5 años para los pacientes que accedieron a cirugía R0 ( $n=51)$, lo que no solo se halla en línea con las series publicadas sino que reafirma el concepto de cirugía R0 como objetivo principal a lograr en estos casos. 


\section{Conclusiones}

Las resecciones multiviscerales en el contexto de Recaídas Locoregionales de $\mathrm{Ca}$ de Recto $\mathrm{y}$ sigma son procedimientos factibles en manos de equipos entrenados $y$ contando con la decisión del equipo multidisciplinario para encarar la Cirugía.

La resección $\mathrm{RO}$ es el objetivo principal en estos casos dado que mejora sustancialmente la sobrevida y el pronóstico alejado.

La selección de pacientes es clave para alcanzar objetivos satisfactorios.
Son procedimientos con alta morbimortalidad y potenciales secuelas definitivas en algunos casos mutilantes por lo que la decisión en muchos casos debe ser contrabalanceada contra el pronóstico de la enfermedad y la decisión del paciente.

Las sobrevidas a las que accedimos en los casos de completar la cirugía $\mathrm{RO}$ están en línea con las reportadas en series internacionales.

Se destaca una vez más la importancia de la discusión de estos casos complejos en el marco interdisciplinario de un Comité de Tumores.

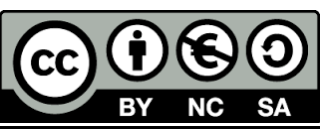

\section{Bibliografía}

1. Lehnert $T$, Methner $M$, Pollok $A$, Schaible A, Hinz U, Herfarth C. Multivisceral Resection for locally advanced primary colon and Rectal cancer. Annals of Surgery. 2002 Feb;235(2):217-25.

2. Moriya $\mathrm{Y}$, Akasu T, Fujita $\mathrm{S}$, Yamamoto S. Total pelvic Exenteration with distal Sacrectomy for fixed recurrent Rectal cancer in the pelvis. Diseases of the Colon \& Rectum. 2004 Dec;47(12):2047-54.

3. Yun J-A, Huh JW, Kim HC, Park YA, Cho YB, Yun SH, Lee WY, Chun H-K. Local recurrence after curative resection for rectal carcinoma. Medicine. 2016 Jul;95(27):e3942.

4. Rizzuto A, Palaia I, Vescio G, Serra $R$, Malanga D, Sacco R. Multivisceral resection for occlusive colorectal cancer: Is it justified? International Journal of Surgery. 2016 Sep;33:S1427.

5. Balyasnikova S, Brown G. Optimal imaging strategies for Rectal cancer staging and ongoing management.
Current Treatment Options in Oncology. 2016 Jun;17(6).

6. Renehan AG. Corrigendum to 'Techniques and outcome of surgery for locally advanced and local recurrent Rectal Cancer' [Clinical oncology 28 (2016) 103-115]. Clinical Oncology. 2016 Aug;28(8):e68.

7. Cameron MG, Kersten C, Vistad I, van Helvoirt R, Weyde $K$, Undseth $C$, Mjaaland I, Skovlund E, Fosså SD, Guren MG. Palliative pelvic radiotherapy for symptomatic rectal cancer - a prospective multicenter study. Acta Oncologica. 2016 Jun 22;55(12):1400-7.

8. Larkin JO, OConnell PR. Multivisceral Resection for T4 or recurrent Colorectal cancer. Digestive Diseases. 2012;30(s2):96-101.

9. Heriot A, Lynch A, Warrier S. Surgery for locally recurrent Rectal cancer: Tips, tricks, and pitfalls. Clinics in Colon and Rectal Surgery. 2016 May 26;29(02):114-22. 